\title{
Superhydrophobic polyimide films with high thermal endurance via UV photo-oxidation
}

\author{
H. Y. Gu $u^{1,2}$, Z. Y. $Q i^{1}, W . W u^{3}, Y$. Zeng $^{3}$, L. X. Song ${ }^{1 *}$ \\ ${ }^{1}$ Key Laboratory of Inorganic Coating Materials, Shanghai Institute of Ceramics, Chinese Academy of Sciences, 1295 \\ Dingxi Road, 200050 Shanghai, China \\ ${ }^{2}$ University of Chinese Academy of Sciences, 19 Yuquan Road, 100049 Beijing, China \\ ${ }^{3}$ The State Key Laboratory of High Performance Ceramics and Superfine Microstructures, Shanghai Institute of Ceramics, \\ Chinese Academy of Sciences, 1295 Dingxi Road, 200050 Shanghai, China
}

\begin{abstract}
UV photo-oxidation was first applied to fabricate superhydrophobic polyimide (PI) films in combination with fluoroalkylsilane (FAS) modification. During prolonged UV irradiation, commercial flat PI films evolved to form unique micro/nanostructures. Meanwhile, the root mean square (RMS) surface roughness increased from 1.74 to $53.70 \mathrm{~nm}$, leading to a gradual increase of WCA from 105.1 to $159.2^{\circ}$ after FAS treatment. After $72 \mathrm{~h}$ of UV radiation exposure, the micro/nano-structured and FAS-modified PI films exhibited superhydrophobicity with water contact angle (WCA) larger than $150^{\circ}$ and sliding angle (SA) less than $5^{\circ}$. The superhydrophobicity remained even after annealing at $350^{\circ} \mathrm{C}$, which enabled stable utilization under elevated temperature. Stable micro/nanostructures and chemical bonding of FAS were found to contribute to the high thermal endurance. Moreover, the formation mechanism of the superhydrophobic PI films was investigated. The proposed UV photo-oxidation method provides a new route for the industrial fabrication of thermally stable superhydrophobic PI films.
\end{abstract}

Keywords: nanomaterials, superhydrophobic polyimide, UV photo-oxidation, thermal properties

\section{Introduction}

Superhydrophobic surfaces with water contact angle (WCA) larger than $150^{\circ}$ have attracted extensive interest in recent years [1, 2]. Superhydrophobic polymers such as polystyrene (PS) are quite attractive owing to their low cost, light weight and ease of processing [3-5]. However, the performance of these materials deteriorates considerably when exposed to extreme environmental conditions (e.g., heat and tension), which severely limits their applications in harsh environments [6]. Polyimide (PI), a widely used polymer, can overcome these drawbacks due to its unique physical and chemical properties such as excellent thermal stability, high strength and good chemical resistance [7-10]. Therefore, superhydrophobic PI is believed to be more durable for applications in anti-corrosion [11], anti-contamination [12], etc.

Superhydrophobic surfaces should have high surface roughness as well as low surface energy [1]. Generally, $\mathrm{CF}_{3}$-terminated flat surfaces with the lowest surface energy have a WCA not greater than $120^{\circ}$. Larger WCA could only be obtained by altering the surface roughness [13]. Techniques including template [11, 14-16], electrospinning $[6,17,18]$, laser $[12,19]$ and plasma [20-22] are used to construct surface structures for superhydrophobic PI. However, complex operations, novel precursors,

\footnotetext{
${ }^{*}$ Corresponding author, e-mail: sicsonglx@gmail.com

(C) BME-PT
} 
masks or vacuum instruments are usually involved. Hence, facile strategies suitable for industrial fabrication are urgently required.

In this paper, a facile UV photo-oxidation method, which can be easily performed in air at atmospheric pressure, was proposed for the first time to tailor surface morphology of commercial PI films to achieve superhydrophobicity. The evolution of topography and WCA during prolonged UV treatment was investigated. Thermal endurance of the superhydrophobic PI films and formation mechanism of the superhydrophobicity were discussed.

\section{Experimental}

\subsection{Materials}

Pyromellitic dianhydride-oxydianiline (PMDAODA) type PI films (Kapton $100 \mathrm{H}, \mathrm{C}_{22} \mathrm{H}_{10} \mathrm{O}_{5} \mathrm{~N}_{2}$ ) were obtained from Toray DuPont (Tokyo, Japan). 1H,1H,2H,2H-Perfluorodecyltriethoxysilane (FAS, $\left.\mathrm{C}_{10} \mathrm{~F}_{17} \mathrm{H}_{4} \mathrm{Si}\left(\mathrm{OCH}_{2} \mathrm{CH}_{3}\right)_{3}\right)$ was supplied by Alfa Aesar (Ward Hill, MA, USA). Ultra-pure water was prepared using a Milli-Q-Plus system (Millipore, Bedford, MA, USA). Analytical grade ethanol was provided by Sinopharm Chemical Reagent Co., Ltd. (Shanghai, China). Prior to use, all PI films were ultrasonically cleaned for 5 min with purified water followed by ethanol and dried in an oven (DHG9023B, Bilon, Shanghai, China) under $100^{\circ} \mathrm{C}$ for $1 \mathrm{~h}$. Other chemicals were used as received.

\subsection{Fabrication of hydrophobic polyimide}

The method consisted of two main steps, i.e. UV photo-oxidation and consequent fluoroalkylsilane modification (UV/FAS), as shown in Figure 1. UV photo-oxidation was conducted in a chamber (HWS80, BiLon, Shanghai, China) with a mercury-quartz lamp (GPHO287T5VH/4, SunMonde, Shanghai, China) emitting UV light at 254 and $185 \mathrm{~nm}$. The output at $185 \mathrm{~nm}$ was equivalent to about $10 \%$ of that at $254 \mathrm{~nm}$ according to the instructions of the UV lamp. PI films were irradiated $10 \mathrm{~mm}$ from the lamp for $24,48,72$ or $144 \mathrm{~h}$ in air at atmospheric pressure, where the intensity of the $254 \mathrm{~nm} \mathrm{UV}$ light was measured to be $10 \mathrm{~mW} / \mathrm{cm}^{2}$. Fluoroalkylsilane modification was then carried out with FAS in a heated reactor. Specifically, a few droplets (30$50 \mu \mathrm{L})$ of FAS were dispensed at the bottom of a Teflon container $(200 \mathrm{~mL})$ using a micro-adjustable pipette (720000, Dragon, Shanghai, China) while the UV-treated PI films were placed vertically in the

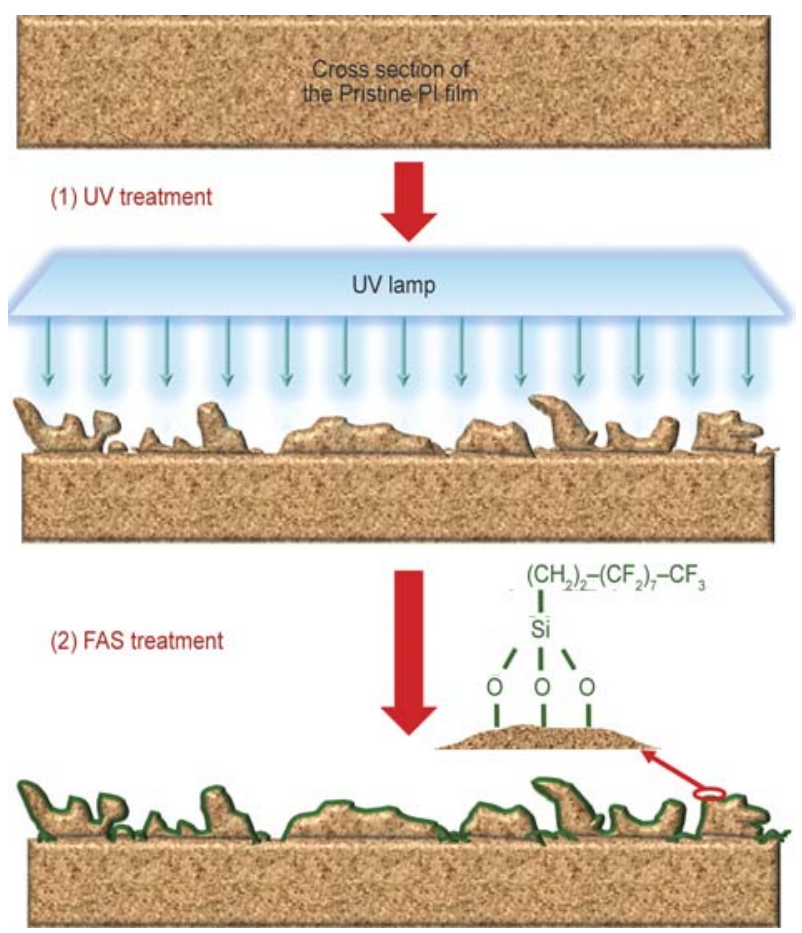

Figure 1. Schematic illustration of the processes involved in the fabrication of hydrophobic PI films

Teflon container, which was further sealed in a stainless steel autoclave. The reactor was then annealed at $120^{\circ} \mathrm{C}$ for $2 \mathrm{~h}$ to enable the vapor of FAS to react with the modified PI films. Finally, the samples were heated at $150^{\circ} \mathrm{C}$ for $3 \mathrm{~h}$ to volatilize unreacted FAS molecules.

\subsection{Thermal treatment}

Thermal endurance of superhydrophobicity of the films was assessed by measuring WCA after annealing for $2 \mathrm{~h}$ under various temperature conditions. A piece of $25 \times 70 \mathrm{~mm}^{2}$ superhydrophobic PI film was fabricated using the method described above $(72 \mathrm{~h}$ of UV photo-oxidation). Then it was cut into seven pieces with identical dimension. One piece is preserved in air at atmospheric pressure without any further treatment, the other six pieces were annealed for $2 \mathrm{~h}$ at $100,150,200,250,300$ and $350^{\circ} \mathrm{C}$, respectively. Then the wettability was studied after the thermally treated films cooled down.

\subsection{Characterization}

The morphology and element distribution were characterized by scanning electron microscopy (SEM) coupled with energy-dispersive X-ray spectroscopy (EDS) on an FEI Magellan 400 microscope (Hillsboro, OR, USA). The surface roughness was analyzed using an atomic force microscope (AFM) 
(Nanoscope V Multimode AFM system, Bruker, Germany) in tapping mode. The surface chemical composition and bonding were examined using an X-ray photoelectron spectroscopy (XPS) (ESCALAB-250 instrument, Thermo VG Scientific, West Sussex, UK). Elemental content was calculated from XPS spectra, based on the peak area of C1s, O1s, F1s and Si2 $p$ and corresponding sensitivity factors using the Avantage software. The static WCA was measured with $5 \mu \mathrm{L}$ water using a contact angle system (SL200B, Solon, Shanghai, China) at ambient temperature. The average WCA value was obtained by measuring the same sample at five different positions. The sliding angle (SA) was determined by slowly tilting the sample stage until the $10 \mu \mathrm{L}$ water droplet starting moving.

\section{Results and discussion}

\subsection{Surface morphology and wettability}

The pristine PI film possessed a flat and slightly hydrophilic surface $\left(\mathrm{WCA}=74.5^{\circ}\right)$ as shown in Figure 2a. To obtain hydrophobicity, FAS treatment was firstly performed on the pristine PI films directly since FAS containing fluoroalkyl chains is commonly used to lower the surface energy $[1,14,16]$. The modified PI surface exhibited hydrophobicity with a WCA of $105.1^{\circ}$ (Figure 2b), which was still far below the criterion value (i.e., $150^{\circ}$ ) of superhydrophobicity. This indicated that the reduction of surface energy was not enough to render the flat PI surface superhydrophobicity and modification of surface structure was required. For this reason, UV irradiation was carried out, in an attempt to change the surface morphology. After $72 \mathrm{~h}$ of $\mathrm{UV}$ radiation exposure, as expected, significant surface roughening was observed and an obvious reduction of WCA to nearly $0^{\circ}$ (Figure 2a, 2c) was measured. Further treatment by FAS after $72 \mathrm{~h}$ of UV irradiation resulted in a WCA of up to $156.8^{\circ}$ (Figure $3 \mathrm{c}$ ) and a low SA less than $5^{\circ}$ (Figure $3 \mathrm{~g}$ ), indicating a superhydrophobic surface. The notable changes of WCA clearly demonstrated the importance of specific surface topography for the wettability. Moreover, UV irradiation was proven to be an effective way to alter the surface topography of PI films.

The evolution of surface morphology and corresponding WCA with UV radiation exposure time was further investigated. As presented in Figure 3a-31, nanoprotrusions emerged, aggregated and interconnected progressively when UV radiation exposure prolonged from 24 to $144 \mathrm{~h}$. After $72 \mathrm{~h}$, micro/ nanostructures with protrusions, ridges and channels were formed on the surfaces, leading to significant surface roughening. The roughness of the surfaces was further quantified by AFM. The root mean square (RMS) roughness and WCA values against UV irradiation time are plotted in Figure 4. As shown in Figure 4, the RMS roughness increased from 1.74 to $53.70 \mathrm{~nm}$ with irradiation time extended from 0 to $144 \mathrm{~h}$. Simultaneously, the WCA increased from 105.1 to $159.2^{\circ}$ due to the surface roughening. Additionally, water drops $(10 \mu \mathrm{L})$ were found to readily roll off the resultant hydrophobic PI films when UV photo-oxidation time exceeded $72 \mathrm{~h}$. Therefore, the wettability of PI films could be tuned by changing the UV irradiation time and $72 \mathrm{~h}$ of exposure time was enough to achieve superhydrophobicity. Considering possible adverse effects on the substrates during UV radiation exposure, minimum UV irradiation time should be used, provided that the required superhydrophobicity is achieved.

\subsection{Thermal endurance}

High thermal stability is a significant advantage of PI over other polymers. It is reasonable to expect the as-prepared superhydrophobic PI films to be thermally stable. In order to validate the hypothesis, we invested the thermal endurance of superhydropho-

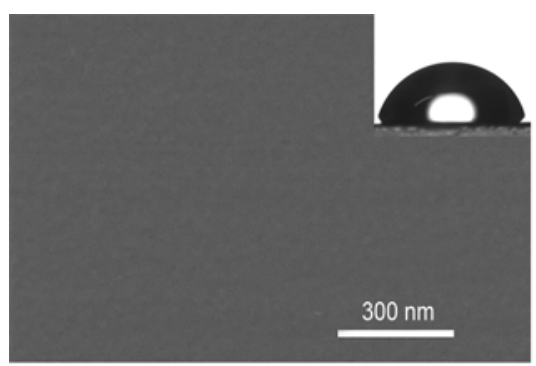

a)

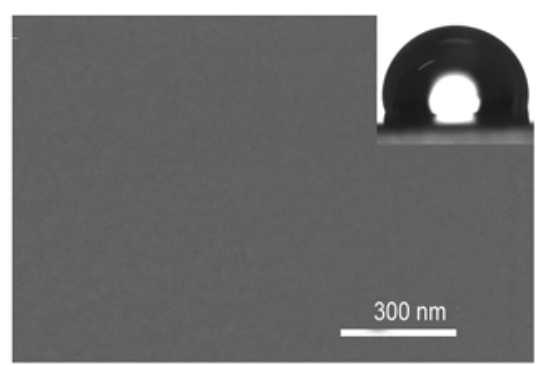

b)

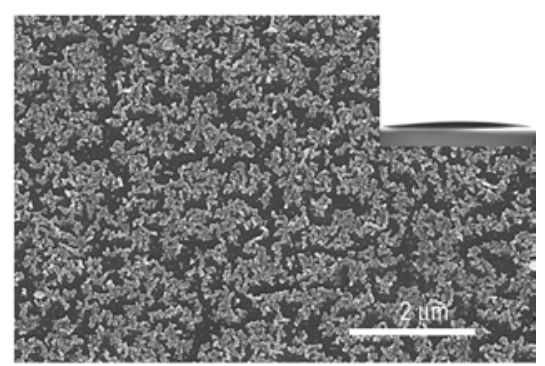

c)

Figure 2. SEM images of pristine (a), FAS (b) and UV (c) treated PI films. The insets are optical images of water droplets on corresponding surfaces. 

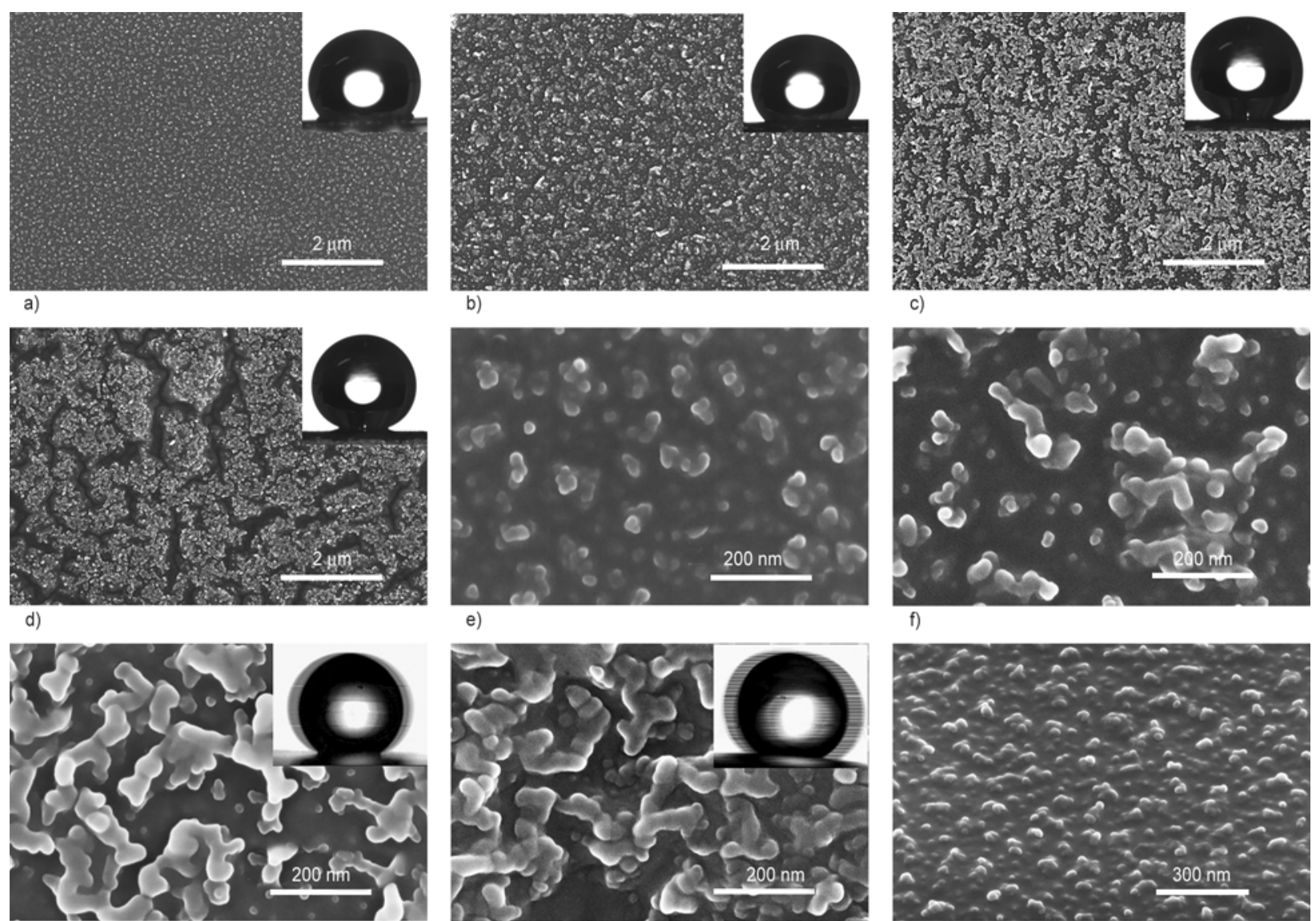

g)

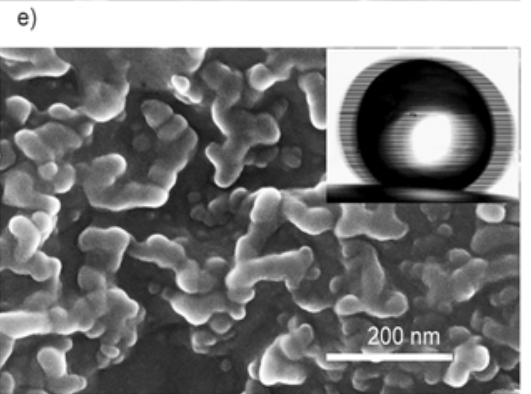

h)

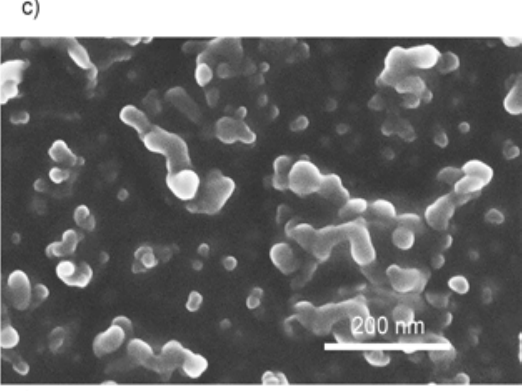

\section{f)}
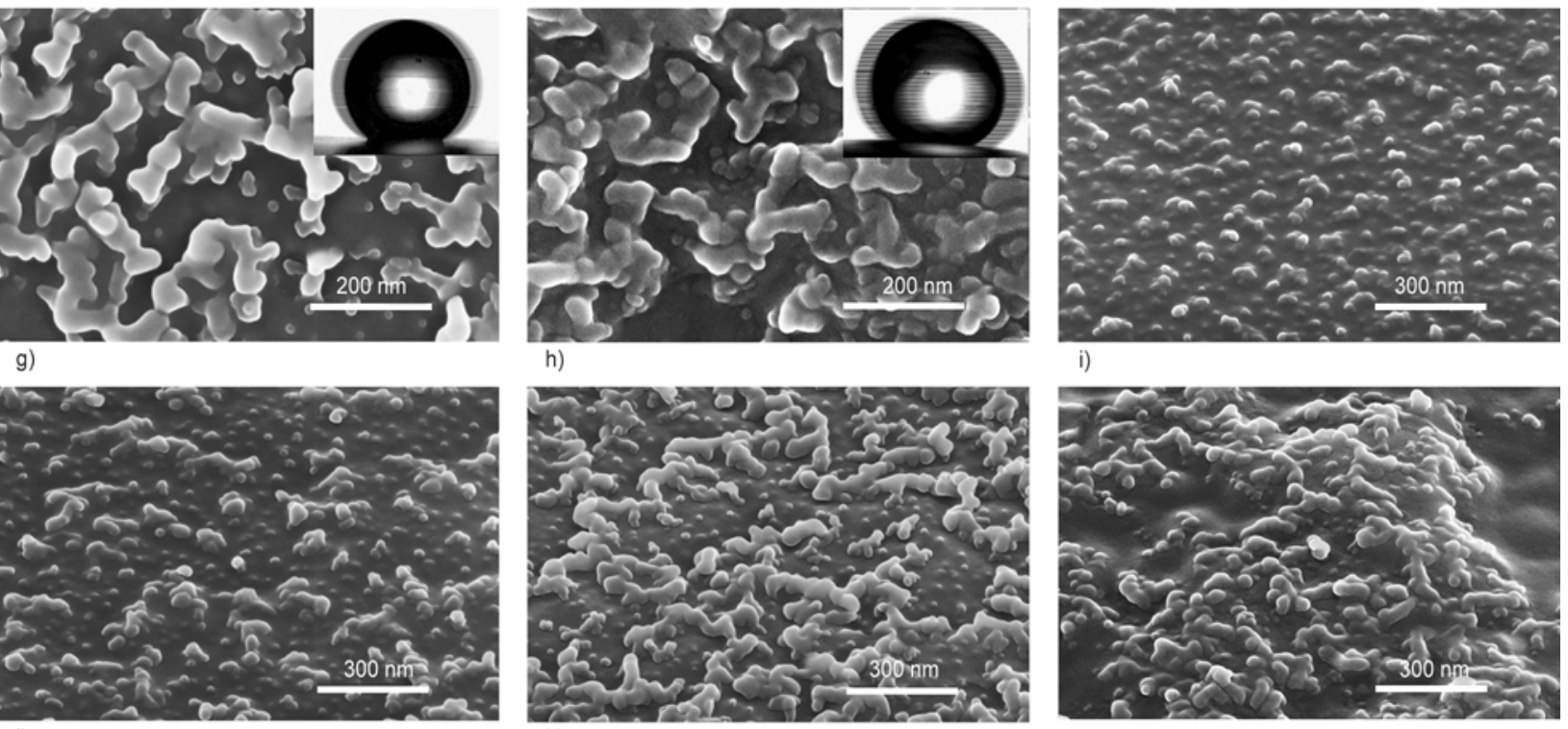

i)

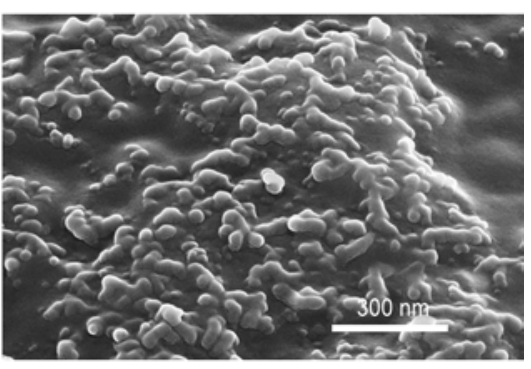

Figure 3. $0^{\circ}(\mathrm{a}-\mathrm{h})$ and $45^{\circ}(\mathrm{i}-\mathrm{l})$ tilt SEM images of UV/FAS treated PI films. The UV treatment periods are $24 \mathrm{~h}(\mathrm{a}, \mathrm{e}, \mathrm{i})$, $48 \mathrm{~h}(\mathrm{~b}, \mathrm{f}, \mathrm{j}), 72 \mathrm{~h}(\mathrm{c}, \mathrm{g}, \mathrm{k})$ and $144 \mathrm{~h}(\mathrm{~d}, \mathrm{~h}, \mathrm{l})$, respectively. The insets are optical images of static water droplets (a-d) on corresponding horizontal surfaces and water droplets $(g, h)$ sliding on slightly tilted surfaces.

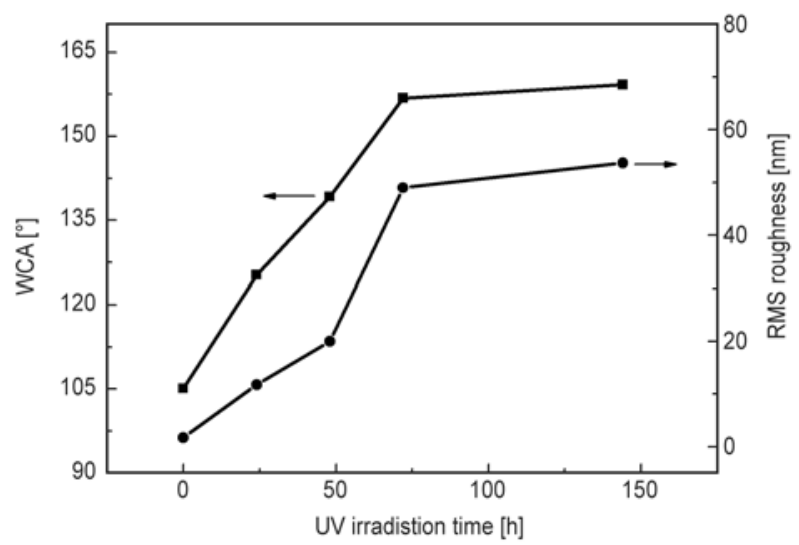

Figure 4. WCA and RMS roughness plotted against UV irradiation time

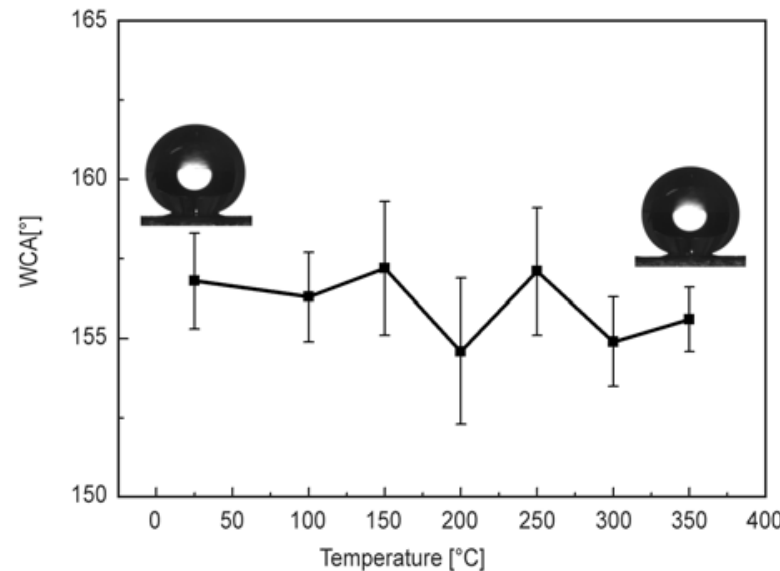

Figure 5. WCA plotted against thermal treatment temperature 


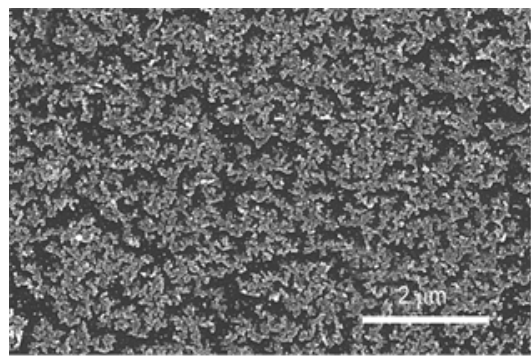

a)

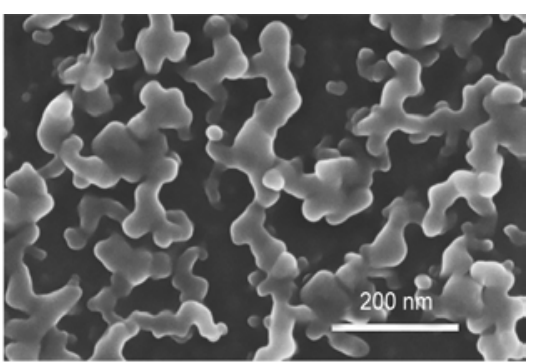

b)

Figure 6. SEM images of annealed $\left(350^{\circ} \mathrm{C}\right)$ superhydrophobic PI films using different magnifications

bicity by annealing the UV (72 h)/FAS-treated PI films below the glass transition temperature $\left(T_{\mathrm{g}}\right)$ of PI [7]. Figure 5 presents the WCA values of samples annealed at corresponding temperatures $(100$ $350^{\circ} \mathrm{C}$ ) for $2 \mathrm{~h}$. Long time thermal treatment seemed to have little effect on the WCA. Only a slight fluc-

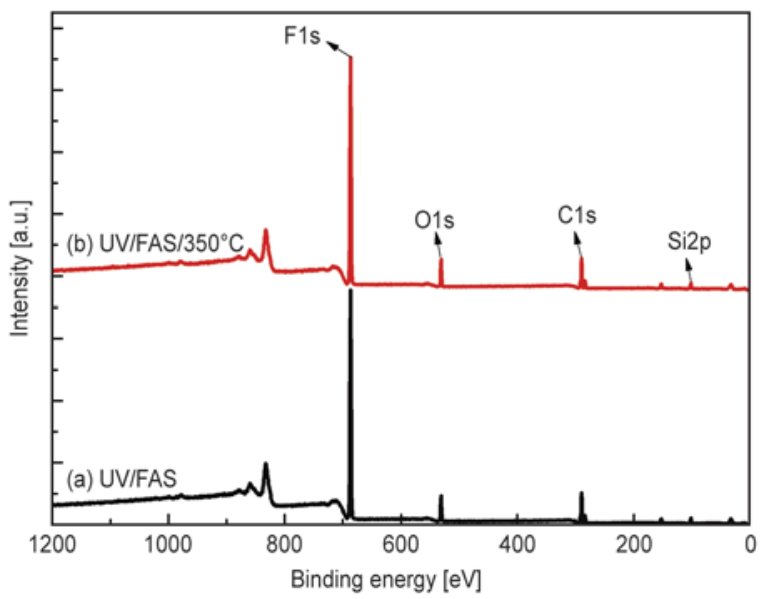

Figure 7. XPS spectra of UV (72 h)/FAS-treated PI before (a) and after (b) annealing at $350^{\circ} \mathrm{C}$ tuation of WCA around $155^{\circ}$ was observed. The SA was also below $5^{\circ}$ for all samples investigated in Figure 5. The WCA larger than $150^{\circ}$ and SA less than $5^{\circ}$ after annealing of the superhydrophobic films demonstrated the highly stable superhydrophobicity. To the contrary, most well-known polymers such as PS cannot sustain their superhydrophobicity at high temperature due to unstable micro/nanostructures [6]. The remained superhydrophobicity of PI films can be ascribed to the thermally stable surface topography and composition. This is confirmed by the well-preserved micro/nanostructures of $350^{\circ} \mathrm{C}$ annealed UV (72 h)/FAS-treated PI (Figure 6) and almost unchanged surface elemental content (relative intensity of peaks) as determined by XPS shown in Figure 7. Specifically, the amount of F changed little from 53.4 at.\% (before annealing) to 52.1 at.\% (after annealing). In addition, the distribution of elements for UV (72 h)/FAS-treated PI films during annealing at $350^{\circ} \mathrm{C}$ were further investigated. As shown in Figure 8, F and Si (derived

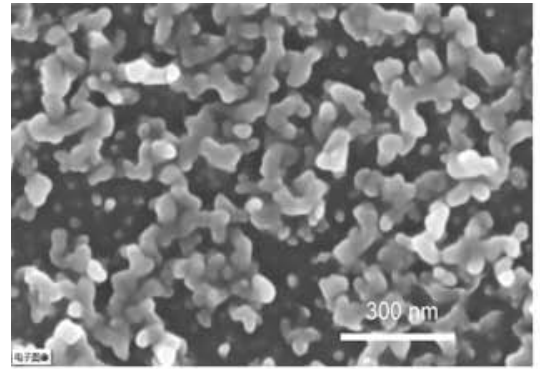

a)

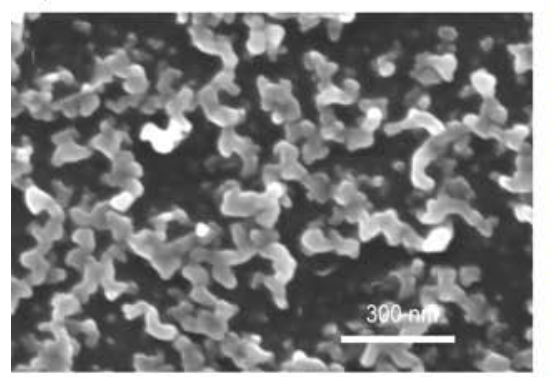

d)

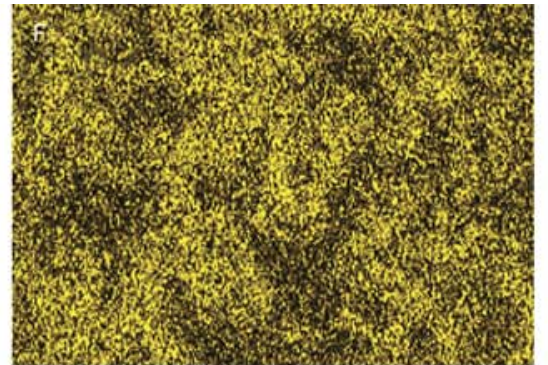

b)

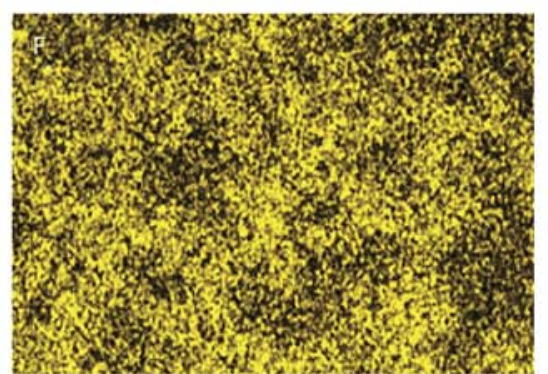

e)

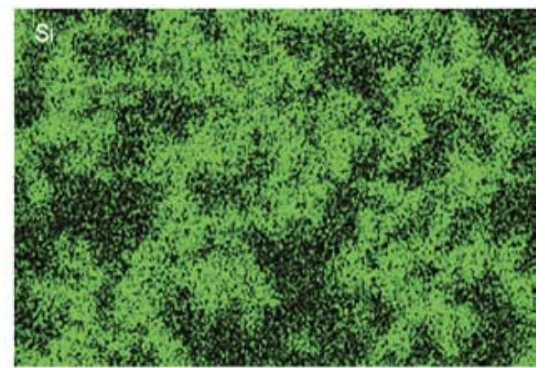

c)

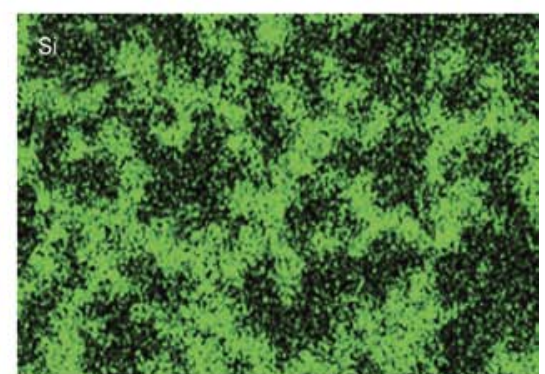

f)

Figure 8. FESEM-EDS mappings for $\mathrm{F}$ and $\mathrm{Si}$ of $\mathrm{UV}(72 \mathrm{~h}) / \mathrm{FAS}$-treated PI before $(\mathrm{a}-\mathrm{c})$ and after $(\mathrm{d}-\mathrm{f})$ annealing at $350^{\circ} \mathrm{C}$ 
from FAS) presented the coincident distribution patterns with the corresponding SEM images both before and after heat treatment at $350^{\circ} \mathrm{C}$. Little change in the distribution of $\mathrm{F}$ and $\mathrm{Si}$ was found after annealing. It is worth pointing out the non-uniform distribution of $\mathrm{F}$ and $\mathrm{Si}$ revealed by the FESEM-EDS mappings. It is clearly shown that FAS distributed preferentially at the ridges on both the as-prepared and the annealed films. FAS modification was proven to be a chemical process that was more likely to occur at the ridges. The strong chemical bonding of FAS on the surface also contributed to the stable superhydrophobicity.

\subsection{Formation mechanism}

Based on the previous discussion, we propose a possible formation mechanism for the superhydrophobic PI films, which was illustrated in Figure 9. During the UV irradiation, low-molecular-weight polymer chains were firstly formed on the film surfaces because of the UV photo-oxidation induced chain scission and recombination [23]. Meanwhile, active oxygens such as atomic oxygens generated during UV radiation also reacted with PI [23]. These oxidized polymer chains had a tendency to aggregate into nanoprotrusions due to high energy dangling bonds and polar groups. Driven by the different interface free energy levels between irradiated surface and underlying PI, the nanoprotrusions further interconnected to form ridges through surface dynamics (e.g., etching, migration and redeposition) [24]. The evolution of the nanoprotrusions with prolonged UV irradiation resulted in the increase of surface roughness, as shown in Figure 3 and Figure 4. Consequently, unique micro/nanostructures with the RMS roughness larger than $49.00 \mathrm{~nm}$ were constructed. Simultaneously, oxidized polymer chains generated hydrophilic groups in the presence of water molecules in air on UV-treated surfaces (e.g., $\mathrm{OH}$ groups) [23], especially at the ridges since more oxidized polymer chains aggregated there. These groups reacted with FAS (fluoroalkyl silanization). Thus, fluoroalkyl chains were firmly grafted onto the UV-irradiated surfaces, resulting in stable superhydrophobicity on the PI films.

\section{Conclusions}

In summary, UV irradiation was used for the first time to prepare superhydrophobic PI films. Unique micro/nanostructures consisting of protrusions, ridges and channels were constructed on the PI surfaces after $72 \mathrm{~h}$ of UV photo-oxidation. The surface roughening led to continuously increased hydrophobicity after further fluoroalkyl silanization. Superhydrophobic surfaces with WCA larger than $156.8^{\circ}$ and SA less than $5^{\circ}$ were achieved for the micro/nano-struc-

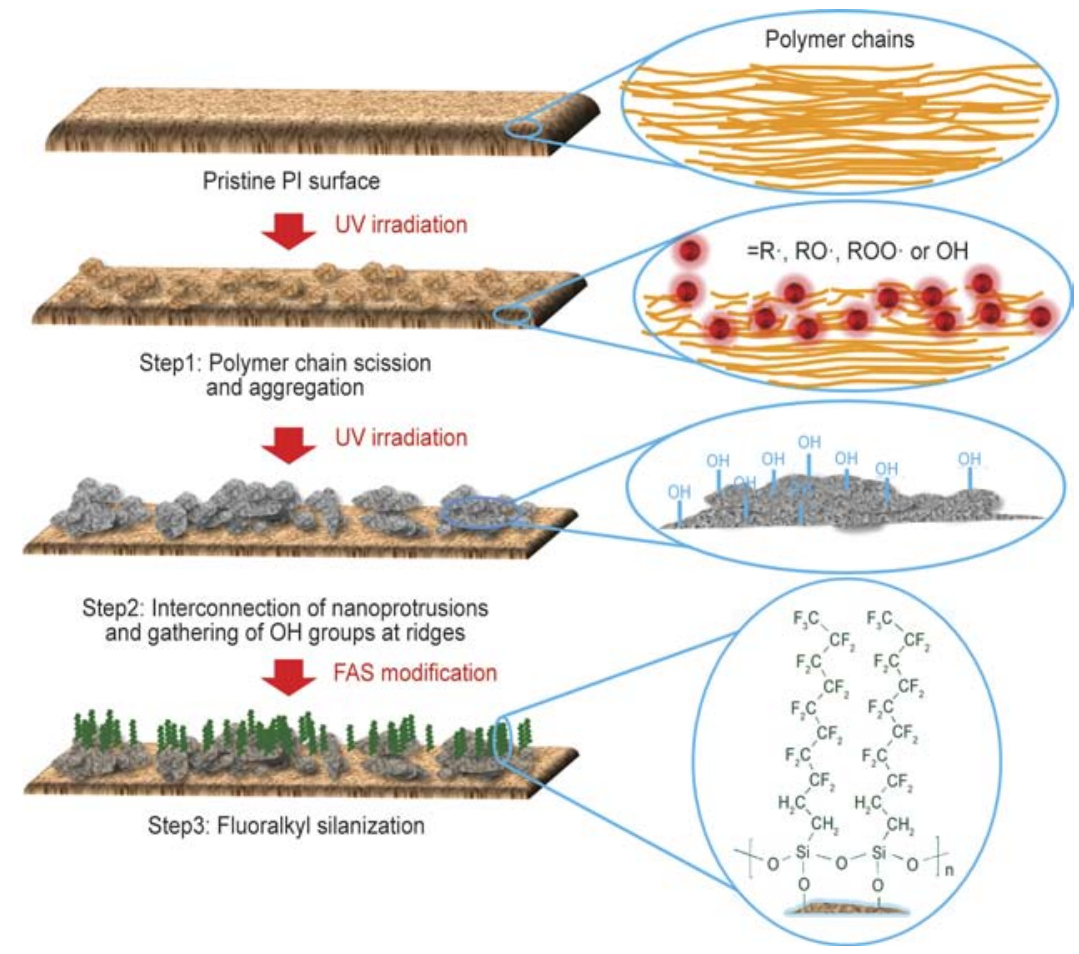

Figure 9. Proposed mechanism for the formation of superhydrophobic PI films 
tured and FAS-modified PI films, indicating that the water drops will easily roll off a slightly tilted substrate. The superhydrophobicity remained even after annealing under $350^{\circ} \mathrm{C}$. Stable micro/nanostructures and chemical bonding of FAS were responsible for the high thermal endurance of superhydrophobicity. These heat endurable superhydrophobic PI films would find lots of practical applications in anti-corrosion, anti-contamination and anti-icing systems.

\section{References}

[1] Liu K., Tian Y., Jiang L.: Bio-inspired superoleophobic and smart materials: Design, fabrication, and application. Progress in Materials Science, 58, 503-564 (2013). DOI: $10.1016 /$ j.pmatsci.2012.11.001

[2] Liu K., Jiang L.: Bio-inspired self-cleaning surfaces. Annual Review of Materials Research, 42, 231-263 (2012).

DOI: 10.1146/annurev-matsci-070511-155046

[3] Darmanin T., de Givenchy E. T., Amigoni S., Guittard F.: Superhydrophobic surfaces by electrochemical processes. Advanced Materials, 25, 1378-1394 (2013). DOI: $\underline{10.1002 / \mathrm{adma} .201204300}$

[4] Wolfs M., Darmanin T., Guittard F.: Superhydrophobic fibrous polymers. Polymer Reviews, 53, 460-505 (2013).

DOI: $10.1080 / 15583724.2013 .808666$

[5] Sahoo B. N., Kandasubramanian B., Sabarish B.: Controlled anisotropic wetting behaviour of multi-scale slippery surface structure of non fluoro polymer composite. Express Polymer Letters, 7, 900-909 (2013). DOI: 10.3144/expresspolymlett.2013.88

[6] Gong G., Wu J., Liu J., Sun N., Zhao Y., Jiang L.: Bioinspired adhesive superhydrophobic polyimide mat with high thermal stability. Journal of Materials Chemistry, 22, 8257-8262 (2012).

DOI: $10.1039 / \mathrm{c} 2 \mathrm{jm} 16503 \mathrm{a}$

[7] Ghosh M. K., Mittal K. L.: Polyimides: Fundamentals and applications. Marcel Dekker, New York (1996).

[8] Zhu Y-Q., Zhao P-Q., Cai X-D., Meng W-D., Qing FL.: Synthesis and characterization of novel fluorinated polyimides derived from bis[4-(4'-aminophenoxy) phenyl]-3,5-bis(trifluoromethyl)phenyl phosphine oxide. Polymer, 48, 3116-3124 (2007).

DOI: 10.1016/j.polymer.2007.03.057

[9] Han Y., Fang X-Z., Zuo X-X.: Melt processable homoand copolyimides with high thermo-oxidative stability as derived from mixed thioetherdiphthalic anhydride isomers. Express Polymer Letters, 4, 712-722 (2010). DOI: $10.3144 /$ expresspolymlett.2010.86

[10] Uğur M. H., Toker R. D., Kayaman-Apohan N., Güngör A.: Preparation and characterization of novel thermoset polyimide and polyimide-peo doped with $\mathrm{LiCF}_{3} \mathrm{SO}_{3}$. Express Polymer Letters, 8, 123-132 (2014). DOI: $10.3144 /$ expresspolymlett.2014.15
[11] Chang K-C., Lu H-I., Peng C-W., Lai M-C., Hsu S-C., Hsu M-H., Tsai Y-K., Chang C-H., Hung W-I., Wei Y., Yeh J-M.: Nanocasting technique to prepare lotus-leaflike superhydrophobic electroactive polyimide as advanced anticorrosive coatings. ACS Applied Materials and Interfaces, 5, 1460-1467 (2013).

DOI: $10.1021 / \mathrm{am} 3029377$

[12] Wohl C. J., Belcher M. A., Chen L., Connell J. W.: Laser ablative patterning of copoly(imide siloxane)s generating superhydrophobic surfaces. Langmuir, 26, 11469-11478 (2010).

DOI: $10.1021 / 1 \mathrm{a} 100958 \mathrm{r}$

[13] Nishino T., Meguro M., Nakamae K., Matsushita M., Ueda Y.: The lowest surface free energy based on $-\mathrm{CF}_{3}$ alignment. Langmuir, 15, 4321-4323 (1999).

DOI: $10.1021 / 1 \mathrm{a} 981727 \mathrm{~s}$

[14] Zhao Y., Li M., Lu Q., Shi Z.: Superhydrophobic polyimide films with a hierarchical topography: Combined replica molding and layer-by-layer assembly. Langmuir, 24, 12651-12657 (2008).

DOI: $10.1021 / 1 \mathrm{a} 8024364$

[15] Zhu S., Li Y., Zhang J., Lü C., Dai X., Jia F., Gao H., Yang B.: Biomimetic polyimide nanotube arrays with slippery or sticky superhydrophobicity. Journal of Colloid and Interface Science, 344, 541-546 (2010). DOI: $10.1016 /$ j.jcis.2009.12.047

[16] Liu K., Du J., Wu J., Jiang L.: Superhydrophobic gecko feet with high adhesive forces towards water and their bio-inspired materials. Nanoscale, 4, 768-772 (2012). DOI: $10.1039 / \mathrm{c} 1 \mathrm{nr} 11369 \mathrm{k}$

[17] Weng C-J., Jhuo Y-S., Chang C-H., Feng C-F., Peng C-W., Dai C-F., Yeh J-M., Wei Y.: A smart surface prepared using the switchable superhydrophobicity of neat electrospun intrinsically electroactive polyimide fiber mats. Soft Matter, 7, 10313-10318 (2011). DOI: $10.1039 / \mathrm{c} 1 \mathrm{sm} 06097 \mathrm{j}$

[18] Park J. Y., Oh K. O., Won J. C., Han H., Jung H. M., Kim Y. S.: Facile fabrication of superhydrophobic coatings with polyimide particles using a reactive electrospraying process. Journal of Materials Chemistry, 22, 16005-16010 (2012).

DOI: $10.1039 / \mathrm{c} 2 \mathrm{jm} 32210 \mathrm{~b}$

[19] Oliveira V., Nunes B., Vilar R.: Wetting response of $\mathrm{KrF}$ laser ablated polyimide surfaces. Nuclear Instruments and Methods in Physics Research Section B: Beam Interactions with Materials and Atoms, 268, 1626-1630 (2010).

DOI: $10.1016 /$ j.nimb.2010.03.006

[20] Scheen G., Ziouche K., Bougrioua Z., Godts P., Leclercq D., Lasri T.: Simultaneous fabrication of superhydrophobic and superhydrophilic polyimide surfaces with low hysteresis. Langmuir, 27, 6490-6495 (2011). DOI: $10.1021 / 1 \mathrm{a} 1050805$ 
[21] Barshilia H. C., Ananth A., Gupta N., Anandan C.: Superhydrophobic nanostructured Kapton ${ }^{\circledR}$ surfaces fabricated through $\mathrm{Ar}+\mathrm{O}_{2}$ plasma treatment: Effects of different environments on wetting behaviour. Applied Surface Science, 268, 464-471 (2013).

DOI: 10.1016/j.apsusc.2012.12.130

[22] Wohl C. J., Belcher M. A., Ghose S., Connell J. W.: Modification of the surface properties of polyimide films using polyhedral oligomeric silsesquioxane deposition and oxygen plasma exposure. Applied Surface Science, 255, 8135-8144 (2009).

DOI: $10.1016 /$ j.apsusc. 2009.05.030
[23] Sener U., Parekh B., Entenberg A., Debies T., Takács G. A.: Surface modification of poly(biphenyl dianhydride-para-phenylene diamine) (BPDA-PDA) polyimide by UV photo-oxidation. Journal of Adhesion Science and Technology, 20, 319-334 (2006). DOI: $10.1163 / 156856106776381776$

[24] Yun J., Bae T-S., Kwon J-D., Lee S., Lee G-H.: Antireflective silica nanoparticle array directly deposited on flexible polymer substrates by chemical vapor deposition. Nanoscale, 4, 7221-7230 (2012).

DOI: $10.1039 / \mathrm{c} 2 \mathrm{nr} 32381 \mathrm{~h}$ 